\title{
Obstructive Shock
}

\author{
Carrie Morgan ${ }^{1}$ and Derek S. Wheeler ${ }^{*}, 2,3$ \\ ${ }^{I}$ Division of Critical Care Medicine, Blair E. Batson Children's Hospital, Jackson, MS, USA \\ ${ }^{2}$ Division of Critical Care Medicine, Cincinnati Children's Hospital Medical Center, Cincinnati, OH, USA \\ ${ }^{3}$ Department of Pediatrics, University of Cincinnati, College of Medicine, USA
}

\begin{abstract}
Obstructive shock is a less common, but important cause of shock in critically ill infants and children. It is caused by mechanical obstruction of blood flow to and/or from the heart and causes can include a tension pneumothorax, cardiac tamponade, pulmonary embolus, or cardiac defects resulting in left-sided outflow tract obstruction. Each of these can result in similar physiology with poor perfusion and oxygen delivery to the body, though the diagnosis and treatment are unique to each process and thus described individually.
\end{abstract}

Keywords: Obstructive shock, pneumothorax, cardiac tamponade, pulmonary embolus, left-sided obstructive heart lesion.

\section{INTRODUCTION}

Hinshaw and Cox developed a classification of shock based upon the different hemodynamic profiles of shock states in 1972. These categories included hypovolemic shock, distributive shock, cardiogenic shock, and extracardiac obstructive shock. The latter was described as involving obstruction to flow in the cardiovascular circuit and characterized by impairment of diastolic filling or excessive afterload [1]. The consequent obstruction of blood flow into or out of the heart causes a decrease in cardiac output, and hence inadequate oxygen delivery, which is manifest by the classic signs and symptoms of the shock state. Obstructive shock is rare in pediatrics, though the most common causes generally include tension pneumothorax, cardiac tamponade, and pulmonary embolism. Also included in this category physiologically, and more specific to pediatrics, are congenital heart lesions characterized by left ventricular outflow tract obstruction, including critical aortic stenosis, coarctation of the aorta, interrupted aortic arch, and hypoplastic left heart syndrome. Herein, we will briefly review the major causes of obstructive shock found in children.

\section{TENSION PNEUMOTHORAX}

A pneumothorax is defined as the accumulation of air in the pleural space, a cavity that is normally filled with a small amount of pleural fluid. It can be spontaneous (more common in adolescent males) or secondary to underlying lung pathology, such as trauma (both penetrating and blunt trauma), asthma, cystic fibrosis, and pneumonia [2]. Also included in this subcategory are iatrogenic causes such as barotrauma during positive pressure ventilation or during

*Address correspondence to this author at the Division of Critical Care Medicine, Cincinnati Children's Hospital Medical Center, 3333 Burnet Avenue, Cincinnati, OH 45229-3039, USA; Tel: (513) 636-4259;

Fax: (513) 636-4267; E-mail: Derek.wheeler@cchmc.org placement of central venous catheters in the chest vessels. The incidence of secondary pneumothorax in pediatric patients is not well described, however, in critically ill children requiring mechanical ventilation it is reported to be $4-15 \%[2,3]$. Notably, the incidence of secondary pneumothorax in mechanically ventilated pediatric patients has declined markedly since the introduction of protective lung strategies [4].

Pneumothoraces can be well tolerated in some patients, though signs and symptoms of obstructive shock can develop if the pneumothorax is under tension. In this scenario, the air in the pleural space continues to collect under a one-way or ball valve effect, such that air enters during inhalation, but cannot exit during exhalation. Eventually, enough air accumulates such that the intrathoracic pressure of the affected hemi-thorax equilibrates with atmospheric pressure, leading to complete lung collapse or atelectasis. Air under tension also causes a shifting of the mediastinum, compression and total collapse of the lung and great vessels, thereby compromising both cardiovascular and respiratory function [5]. Studies in animal models have shown that the early clinical features of a tension pneumothorax include hypoxemia, tachycardia, and respiratory distress due to compression and collapse of lung segments. As mechanical compromise of venous structures develops, there is a drastic and profound reduction in venous return to the heart as well, clinically manifested by symptoms of shock and poor perfusion. Thus, overt hypotension may be a late sign. Complete occlusive mechanical compression is suggested by equalization of the Mean Intrathoracic Pressure (MIP) and Central Venous Pressure (CVP), which is a very late event and results in cardiovascular collapse [6]. Treatment of a tension pneumothorax requires emergent needle decompression, usually performed by placing a sterile needle in the second intercostal space along the midclavicular line. Definitive treatment requires thoracostomy tube placement $[3,5]$. 


\section{CARDIAC TAMPONADE}

The pericardial sac around the heart is relatively noncompliant, and the accumulation of even small amounts of fluid can be sufficient to produce cardiac tamponade physiology. While acute pericardial fluid changes are usually symptomatic, the chronic accumulation of fluid may occur with little to no hemodynamic derangements, as the pericardium slowly stretches to accommodate the excess volume over time [7]. Pericardial effusions can develop as a result of any type of pericardial inflammation (i.e., pericarditis), causing a range of physiologic perturbations along the spectrum of minor flu-like symptoms (i.e., manifestations of the pericarditis itself) to a life-threatening state characterized by cardiac tamponade and obstructive shock [7]. Historically, the most common cause of pericardial effusions was infectious pericarditis, though a recent review suggests that idiopathic and neoplastic causes are much more frequent due to the success of childhood vaccinations [8]. Other common causes include postpericardiotomy syndrome (following cardiac surgery for congenital heart disease) and trauma, most often causing hemopericardium. Effusions may also develop as a result of a central line that erodes through the thin wall of the right atrium, a phenomenon that appears primarily limited to neonates and young infants [9].

The pathophysiology of cardiac tamponade is welldescribed. Briefly, increased intrapericardial pressure limits venous return to the heart and causes right ventricular compression. There is a progressive decline in right ventricular end-diastolic volume as diastolic filling lessens, worsening cardiac output. In severe tamponade, venous return during inspiration into the compressed right ventricle bows the interventricular septum into the left ventricle, further diminishing systemic cardiac output. As pericardial pressure increases and surpasses ventricular end-diastolic pressure, the ventricular volumes grow smaller and smaller and cardiac output worsens [10].

Tamponade is a clinical diagnosis and classically, patients with critical cardiac tamponade present with Beck's triad of symptoms including hypotension, quiet ("muffled") heart sounds, and raised jugular venous pressure [7, 10,11]. Patients may present with dyspnea, compensatory tachycardia, and poor perfusion. On auscultation, a friction rub and distant heart sounds may be present. Pulsus paradoxus, defined as a decline in systolic blood pressure greater than or equal to $10 \mathrm{~mm} \mathrm{Hg}$ during inspiration, results from the inspiratory reduction in pleural pressure that produces a fall in left ventricular output and arterial systolic pressure [5, 7, 12]. An electrocardiogram may show electrical alternans due to the heart swinging within the large effusion. Formal evaluation with an urgent echocardiogram should be performed in those patients with symptoms suspicious for cardiac tamponade. However, emergent management should not wait for echocardiography and is frequently based upon the recognition of tamponade physiology in the appropriate clinical context.

Pericardiocentesis is the lifesaving procedure of choice for children with cardiac tamponade and can safely be done with bedside echocardiographic guidance [11]. Medical stabilization with fluid resuscitation and inotropic support is temporary at best and somewhat controversial as fluid resuscitation may worsen tamponade physiology, especially in children who are either normovolemic or hypervolemic [10]. In the latter scenario, fluid administration will increase intracardiac pressures further, hence increasing intrapericardial pressures and worsening tamponade [12].

\section{PULMONARY EMBOLISM}

Pulmonary embolism (PE) is uncommonly diagnosed in children, making its true incidence difficult to determine. However, the incidence of PE does appear to be on the rise, though this may be due to a heightened index of clinical suspicion and better recognition by pediatric providers. Alternatively, it may be due to the fact that more children are surviving from previously fatal conditions that place them at an increased risk for developing PE, such as congenital heart disease and malignancy. In addition, more children are requiring central venous catheterization for vascular access, a major risk factor for venous thromboembolism (VTE), which can lead to a PE $[13,14]$.

$\mathrm{PE}$ is frequently fatal and difficult to diagnose. In a recent literature review comparing pediatric PE with adult $\mathrm{PE}$, pediatric cases were more often diagnosed at autopsy and were associated with a higher mortality rate than adults [13]. The clinical presentation often is confusing, perhaps compounded by the fact that very few pediatricians have much experience with this disorder. Results of screening tests, such as oxygen saturation, electrocardiography, and chest radiography, may be normal. Thus, a high index of clinical suspicion is necessary. Evaluation should be performed with spiral computed tomography (CT) venography, which is now widely considered the study of choice due to its $>90 \%$ sensitivity and specificity in adults. Ventilation/ Perfusion (V/Q) scans are also available but are more difficult to obtain and to interpret in pediatrics [15].

As a cause of cardiogenic shock, a massive PE has a profound impact upon gas exchange and hemodynamics. Obstruction to flow through the pulmonary artery results in increased dead space ventilation where affected lung segments are ventilated but not perfused, observed clinically as a substantial decrease in the end-tidal $\mathrm{CO}_{2}\left(\mathrm{ETCO}_{2}\right)$ that no longer reflects arterial $\mathrm{PCO}_{2}$. A widened alveolar-arterial gradient (A-a) is present as well. The mechanism for hypoxemia likely involves several mechanisms. In some pediatric patients, an intracardiac right-to-left shunt through a patent foramen ovale may be present and as right atrial pressure increases and eventually exceeds the left atrial pressure, deoxygenated blood can shunt directly into the systemic circulation. In addition, $\mathrm{V} / \mathrm{Q}$ mismatching is compounded by the accompanying fall in cardiac output that results from massive $\mathrm{PE}$, leading to mixed venous desaturation. PE increases the right ventricular (RV) afterload, resulting in an increase in the RV end-diastolic volume (EDV). The increase in RVEDV adversely affects left ventricular hemodynamics through ventricular interdependence. Specifically, the interventricular septum bows into the left ventricle (LV) and impairs diastolic filling, resulting in decreased LV preload and subsequently diminished cardiac output and hypotension $[5,16,17]$. These physiologic phenomenon are manifested by respiratory distress, hypoxia, and decreased cardiac output with signs of shock. 
Treatment of an acute pulmonary embolus in children should begin with initiation of a heparin infusion with or without fibrinolytic agents such as tPA, depending on the child and the extent of the clot. In the resolution period, the child will then warrant at least 3-6 months of anticoagulation with low molecular weight heparin (LMWH) or warfarin [5]. More detailed antithrombotic therapy instruction for pediatric and neonatal patients can be found in the clinical practice guidelines for anti-thrombotic therapy published in 2008 [18].

\section{LEFT-SIDED CARDIAC OBSTRUCTION}

Obstructive shock may also present in specific cardiac lesions that have ductal-dependent systemic blood flow. There are several left-sided heart lesions that are dependent upon ductal blood flow through a patent ductus arteriosus (PDA) to maintain systemic circulation, these include coarctation of the aorta, critical aortic valve stenosis, interrupted aortic arch, and hypoplastic left heart syndrome. As the PDA closes after birth, systemic blood flow decreases and the pulmonary system receives more and more blood flow. This results in the development of heart failure with respiratory distress due to pulmonary edema, impaired systemic perfusion with acidosis, and eventually circulatory collapse. These anatomic defects require prompt recognition in the neonatal period, if not prenatally diagnosed, and the treatment depends on circulatory and respiratory support including mechanical ventilation and inotropic support of the fluid-overloaded right ventricle, as well as securing ductal patency with prostaglandin E2 infusion. Definitive treatment is later achieved through surgical palliation, discussions of which are outside of the scope of this article $[5,12,19]$.

\section{CONFLICT OF INTEREST}

The authors confirm that this article content has no conflict of interest.

\section{ACKNOWLEDGEMENTS}

Declared none.

\section{REFERENCES}

[1] Cox BG, Hinshaw LB, United States Navy Project THEMIS. University of Oklahoma. Health Sciences Center. The Fundamental mechanisms of shock. In: Lerner B, Hinshaw, Barbara GC, Eds. Proceedings of a symposium held in Oklahoma City; 1971 October 1-2, New York: Plenum Press 1972.

[2] Yarmus L, Feller-Kopman D. Pneumothorax in the critically ill patient. Chest 2012; 141: 1098-105.

[3] Johnson NN, Toledo A, Endom EE. Pneumothorax, pneumomediastinum, and pulmonary embolism. Pediatr Clin North Am 2010; 57: 1357-83.

[4] Miller MP, Sagy M. Pressure characteristics of mechanical ventilation and incidence of pneumothorax before and after the implementation of protective lung strategies in the management of pediatric patients with severe ARDS. Chest 2008; 134: 969-73.

[5] Wheeler DS, Wong HR, Shanley TP. Pediatric critical care medicine : Basic science and clinical evidence. London: Springer 2007.

[6] Barton ED, Rhee P, Hutton KC, Rosen P. The pathophysiology of tension pneumothorax in ventilated swine. J Emerg Med 1997; 15: $147-53$.

[7] Troughton RW, Asher CR, Klein AL. Pericarditis. Lancet 2004; 363: 717-27.

[8] Kuhn B, Peters J, Marx GR, Breitbart RE. Etiology, management, and outcome of pediatric pericardial effusions. Pediatr Cardiol 2008; 29: 90-4.

[9] Weil BR, Ladd AP, Yoder K. Pericardial effusion and cardiac tamponade associated with central venous catheters in children: an uncommon but serious and treatable condition. J Pediatr Surg 2010; 45: 1687-92.

[10] Knudson JD. Diseases of the pericardium. Congenit Heart Dis 2011; 6: 504-13.

[11] Azam S, Hoit BD. Treatment of pericardial disease. Cardiovasc Ther 2011; 29: 308-14.

[12] Keane JF, Lock JE, Fyler DC, Nadas AS. Nadas' pediatric cardiology. $2^{\text {nd }}$ ed. Philadelphia: Saunders Elsevier 2006.

[13] Baird JS, Killinger JS, Kalkbrenner KJ, Bye MR, Schleien CL. Massive pulmonary embolism in children. J Pediatr 2010; 156: 148-51.

[14] Victoria T, Mong A, Altes T, et al. Evaluation of pulmonary embolism in a pediatric population with high clinical suspicion. Pediatr Radiol 2009; 39: 35-41.

[15] McCrory MC, Brady KM, Takemoto C, Tobias JD, Easley RB. Thrombotic disease in critically ill children. Pediatr Crit Care Med 2011; 12: 80-9.

[16] Tapson VF. Acute pulmonary embolism. Cardiol Clin 2004; 22: 353-65, v.

[17] Patocka C, Nemeth J. Pulmonary embolism in pediatrics. J Emerg Med 2012, 42: 105-16.

[18] Monagle P, Chalmers E, Chan A, et al. American College of Chest P. Antithrombotic therapy in neonates and children: American College of Chest Physicians Evidence-Based Clinical Practice Guidelines 8th Ed. Chest 2008; 133(6 Suppl): 887S-968S.

[19] Barron DJ, Kilby MD, Davies B, Wright JG, Jones TJ, Brawn WJ. Hypoplastic left heart syndrome. Lancet 2009; 374: 551-64. 